\title{
The big, the bad, and the exon 11: adjuvant imatinib for all gastro- intestinal stromal tumors or just the ugly?
}

\author{
Roman Groisberg, Vivek Subbiah \\ Department of Investigational Cancer Therapeutics (Phase 1 Program), Division of Cancer Medicine, The University of Texas MD Anderson \\ Cancer Center, Houston, TX, USA \\ Correspondence to: Vivek Subbiah, MD. Department of Investigational Cancer Therapeutics (Phase 1 Program), Division of Cancer Medicine, The \\ University of Texas MD Anderson Cancer Center, 1515 Holcombe Blvd, Unit 455, Houston, Texas 77030, USA. Email: vsubbiah@mdanderson.org. \\ Provenance: This is a Guest Editorial commissioned by Editor-in-Chief Jia-Fu Ji, MD, FACS (Department of Gastrointestinal Surgery, Peking \\ University School of Oncology \& Beijing Cancer Hospital, Beijing, China). \\ Comment on: Joensuu H, Wardelmann E, Sihto H, et al. Effect of KIT and PDGFRA Mutations on Survival in Patients With Gastrointestinal \\ Stromal Tumors Treated With Adjuvant Imatinib: An Exploratory Analysis of a Randomized Clinical Trial. JAMA Oncol 2017;3:602-9.
}

Received: 03 September 2017; Accepted: 18 September 2017; Published: 13 October 2017.

doi: $10.21037 / \operatorname{tgh} .2017 .09 .10$

View this article at: http://dx.doi.org/10.21037/tgh.2017.09.10

Gastrointestinal stromal tumor (GIST), a type of sarcoma, is the most common mesenchymal tumor of the gastrointestinal tract. Because the majority of GIST is driven by KIT proto-oncogene receptor tyrosine kinase (KIT) (encodes KIT protein) (OMIM 164920) and plateletderived growth factor receptor $\alpha$ (PDGFRA) (encodes platelet-derived growth factor receptor $\alpha$ ) (OMIM 173490), it represents a paradigm for targeted therapy in solid tumors. An exploratory analysis of the Scandinavian Sarcoma Group (SSG) XVIII/Arbeitsgemeinschaft Internistische Onkologie (AIO) trial was recently published by Joensuu et al. (1). The parent trial, originally published in 2016, compared 3 years to 1 year of adjuvant imatinib (Gleevec ${ }^{\circledR}$ ) in surgically resected GISTs (2). It established 3 years of adjuvant imatinib as the standard of care for high risk GIST. The authors, hoping to capitalize on lessons learned about the molecular biology of this disease, evaluated the mutation status of patients enrolled in the trial.

Other investigators have previously established the prognostic value of mutations in KIT PDGFRA, showing that deletions are more aggressive than point mutations (3). The authors asked: Does the duration of adjuvant imatinib have an effect on the prognostic power of various mutations?

The analysis was performed retrospectively and is thus limited. To avoid a "fishing expedition" and to maximize power of this retrospective analysis, they focused their efforts on KIT exon 11 mutations in comparison to all others. This was a prudent choice as KIT exon 11 is the most commonly involved mutation site in GIST, and codons 557 and 558 especially are associated with higher mitotic rate and poor prognosis.

The analysis focused on relapse-free survival (RFS) rather than overall survival. This was a deliberate choice since the original trial had RFS as the primary endpoint. It also increased statistical power since there were more RFS events than deaths. The authors chose a two-sided P-value to ensure that differences could be accounted for in either direction.

Unsurprisingly (4), KIT mutations constituted $80.4 \%$ of all samples, $12.6 \%$ were PDGFR $\alpha$, and the remaining $7 \%$ wild type for either gene. Of the KIT mutated patients $54 \%$ had exon 11 deletion or insertion and $74.5 \%$ of those cases involved exon 557 and/or 558. The samples with both codon 557 and 558 deletion had the highest mitotic rate corresponding to the highest risk disease.

The comparison of exon 11 deletions or insertions to all other mutations affirmed that this group had a shorter RFS and highlighted the worst prognosis for double deletion exon 11 codons 557 and 558. This difference was observed in the 1-year imatinib group. However, when the same analysis was performed for the 3 -year group no such differences were observed. The authors concluded that the 
unfavorable prognosis of exon 11 deletions and insertion/ deletions, including those involving codons 557 and/or 588 , could be reversed by extending adjuvant imatinib from 1 year to 3 years. They further suggest that prolonged exposure to imatinib alters the molecular biology of certain GIST subtypes. The same analysis repeated for PDGFR $\alpha$, exon 9, and wild-type GIST samples showed no benefit to the prolonged administration of imatinib.

We commend the authors for undertaking this analysis to shed light on who truly benefits from extended adjuvant imatinib. The price of imatinib has been decried as the poster-child for unsustainable cancer drug prices (5). Beyond the financial strain this puts on individuals, health insurers, and the safety net system this drug comes with a long list of adverse events. Current practice is to put any high risk patient on 3 years of adjuvant imatinib. This analysis suggests that we are grossly over-treating patients. Granted, we are likely excluding most PDGFR $\alpha$ tumors because of their low mitotic rate. However, it is clear that many patients are not benefiting from current practice including patients with exon 9 duplication which has reduced sensitivity to the $400 \mathrm{mg}$ dose of imatinib (6).

One critique of this study is the choice to report the RFS rather than overall survival. This has been validated in colon cancer as an acceptable surrogate marker (7), but the applicability to GIST is not clear. The authors suggest that extended adjuvant imatinib changes the biology of some GISTs. This would have been better demonstrated with overall survival. The original SSG/AIO trial on which this analysis is based reported that 3-years of imatinib resulted in prolonged overall survival. The advantage of this strategy is that a statistically significant result is obtained. The disadvantage is that we are left with a correlation rather than a causation. A prospective trial is needed now to show that 3 (or more) years of adjuvant imatinib improves OS in exon 11 deletions and indels. This trial would of course be difficult to initiate and accrue given the already established standard of care.

The mutational landscape of GIST is becoming increasingly complex. We started with the simple exons 9 and 11, later adding PDGFR a, and exon 11 codon 557 and 558. The rest we classify as wild-type. This wild-type group constitutes $10-15 \%$ of patients and also harbors potentially actionable alterations (4). For example, NF1 and $B R A F$ mutant GIST do not respond to imatinib, but are amenable to other targeted therapies. This study used conventional sequencing of KIT exons $9,11,13$, and 17 as well as PDGFR $\alpha$ exons 12 and 18. This approach covers
$85-90 \%$ of all cases, but can be improved using targeted exome next generation sequencing (NGS). When future studies are designed, the addition of NGS may enhance our understanding of resistance mechanisms, co-occurring mutations, as well as expand our repertoire of therapies (8) for "wild-type" GIST. The use of serial "liquid biopsies" and circulating tumor DNA sequencing can further elucidate the evolution of GIST under pressure from adjuvant imatinib.

In conclusion, we consider this work to be a vital first step in redefining adjuvant therapy for GIST. Not all patients benefit and some may be over treated. In the age of affordable gene sequencing, there is no reason a blanket approach should be applied with adjuvant targeted therapy. This analysis calls for a new biomarker driven prospective trial with randomization to groups based on gene and mutation. If imatinib is used, then an academic or collaborative group trial using public or philanthropic dollars will be needed as it is unlikely that drug companies would sponsor the trial that potentially restricts the indication for their drug. One of the other tyrosine kinase inhibitors in the GIST space may be more interested in funding such an enterprise to move their drug to the frontline or adjuvant setting. A potential trial design is a phase III biomarker stratified design (9) that will allow sufficient power to test such a hypothesis.

\section{Acknowledgements}

None.

\section{Footnote}

Conflicts of Interest: The authors have no conflicts of interest to declare.

\section{References}

1. Joensuu H, Wardelmann E, Sihto H, et al. Effect of KIT and PDGFRA Mutations on Survival in Patients With Gastrointestinal Stromal Tumors Treated With Adjuvant Imatinib: An Exploratory Analysis of a Randomized Clinical Trial. JAMA Oncol 2017;3:602-9.

2. Joensuu H, Eriksson M, Sundby Hall K, et al. Adjuvant Imatinib for High-Risk GI Stromal Tumor: Analysis of a Randomized Trial. J Clin Oncol 2016;34:244-50.

3. Lasota J, Miettinen M. KIT and PDGFRA mutations in gastrointestinal stromal tumors (GISTs). Semin Diagn 
Pathol 2006;23:91-102.

4. Boikos SA, Stratakis CA. The genetic landscape of gastrointestinal stromal tumor lacking KIT and PDGFRA mutations. Endocrine 2014;47:401-8.

5. Experts in Chronic Myeloid Leukemia. The price of drugs for chronic myeloid leukemia (CML) is a reflection of the unsustainable prices of cancer drugs: from the perspective of a large group of CML experts. Blood 2013;121:4439-42.

6. Künstlinger H, Huss S, Merkelbach-Bruse S, et al. Gastrointestinal stromal tumors with KIT exon 9 mutations: Update on genotype-phenotype correlation and

doi: $10.21037 / \operatorname{tgh} .2017 .09 .10$

Cite this article as: Groisberg R, Subbiah V. The big, the bad, and the exon 11: adjuvant imatinib for all gastro-intestinal stromal tumors or just the ugly? Transl Gastroenterol Hepatol 2017;2:81. validation of a high-resolution melting assay for mutational testing. Am J Surg Pathol 2013;37:1648-59.

7. Sargent DJ, Wieand HS, Haller DG, et al. Disease-free survival versus overall survival as a primary end point for adjuvant colon cancer studies: individual patient data from 20,898 patients on 18 randomized trials. J Clin Oncol 2005;23:8664-70.

8. BLU-285, DCC-2618 Show Activity against GIST. Cancer Discov 2017;7:121-2.

9. Simon R. Biomarker based clinical trial design. Chin Clin Oncol 2014;3:39. 